\title{
Enhancing Music Learning with Smart Technologies
}

\author{
Rafael Ramirez \\ Universitat Pompeu Fabra \\ Barcelona, Spain \\ rafael.ramirez@upf.edu
}

\section{Corrado Canepa}

Simone Ghisio

Ksenia Kolykhalova

University of Genoa

Genoa, Italy

corrado@infomus.org

simoneghisio@gmail.com

kolykhalova.ksenia@gmail.com

\section{Maurizio Mancini}

Erica Volta

University of Genoa

Genoa, Italy

maurizio@infomus.org

erica.volta@edu.unige.it

\author{
Gualtiero Volpe \\ University of Genoa \\ Genoa, Italy \\ gualtiero.volpe@unige.it \\ Sergio Giraldo \\ Oscar Mayor \\ Alfonso Perez \\ Universitat Pompeu Fabra \\ Barcelona, Spain \\ sergio.giraldo@upf.edu \\ oscar.mayor@upf.edu \\ alfonso.perez@upf.edu
}

George Waddell
Aaron Williamon
Royal College of Music
London, UK
george.waddell@rcm.ac.uk
aaron.williamon@rcm.ac.uk

Permission to make digital or hard copies of part or all of this work for personal or classroom use is granted without fee provided that copies are not made or distributed for profit or commercial advantage and that copies bear this notice and the full citation on the first page. Copyrights for third-party components of this work must be honored. For all other uses, contact the owner/author(s)

MOCO'18, June 2018, Genova, Italy

(c) 2018 Copyright held by the owner/author(s).

ACM ISBN 123-4567-24-567/08/06 .\$15.00

https://doi.org/10.1145/3212721.3212886 


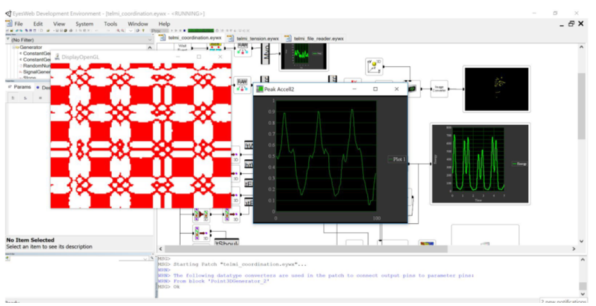

Figure 1: Analysis of coordination, applying Recurrence Quantification Analysis to the kinetic energy of the right wrist.

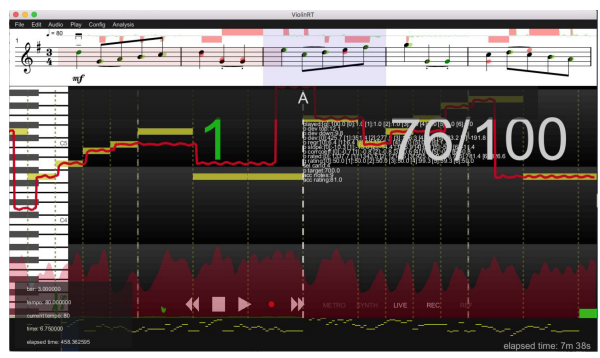

Figure 2: A sample screen-shot of the intonation feedback on Piano Roll Mode.

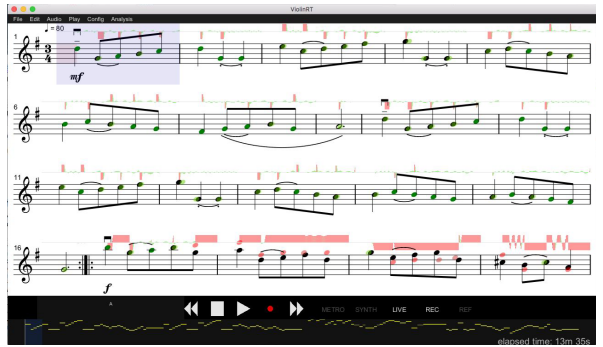

Figure 3: A sample screen-shot of the intonation feedback on Score View Mode.

\section{ABSTRACT}

Learning to play a musical instrument is a difficult task, requiring the development of sophisticated skills. Nowadays, such a learning process is mostly based on the master-apprentice model. Technologies are rarely employed and are usually restricted to audio and video recording and playback. The TELMI (Technology Enhanced Learning of Musical Instrument Performance) Project seeks to design and implement new interaction paradigms for music learning and training based on state-of-the-art multimodal (audio, image, video, and motion) technologies.

The project focuses on the violin as a case study. This practice work is intended as demo, showing to MOCO attendants the results the project obtained along two years of work. The demo simulates a setup at a higher education music institution, where attendants with any level of previous violin experience (and even with no experience at all) are invited to try the technologies themselves, performing basic tests of violin skill and pre-defined exercises under the guidance of the researchers involved in the project.

\section{CCS CONCEPTS}

-Applied computing $\rightarrow$ Sound and music computing; Computer-assisted instruction; Interactive learning environments;

\section{KEYWORDS}

Violin; multimodal interactive systems; technology-enhanced learning; music performance.

\section{ACM Reference Format:}

Rafael Ramirez, Gualtiero Volpe, Corrado Canepa, Simone Ghisio, Ksenia Kolykhalova, Sergio Giraldo, Oscar Mayor, Alfonso Perez, Maurizio Mancini, Erica Volta, George Waddell, and Aaron Williamon. 2018. Enhancing Music Learning with Smart Technologies. In Proceedings of 5th International Conference on Movement Computing (MOCO'18). ACM, New York, NY, USA, 4 pages. https://doi.org/10.475/123_4

\section{INTRODUCTION}

Master violin playing is a high complex activity, requiring to both be proficient in its technique and on potentially a huge repertoire of techniques exercises and musical composition. Nonetheless, in violin learning, as in any other domain, the basic learning process by which violinists acquire new skills and knowledge are based on incorporating principles of effortful practice, systematic reflection, a good planning, and so on. It also requires the development of a finely tuned sensory-motor system driven by auditory, visual, and haptic feedback. Violinists learn their instruments in specific contexts and have a vast set of resources at their disposal. In these situations, however, musicians also face major challenges, including communicative barriers between student and teacher, the competitive nature 
of advanced musical study, and a restrictive environment based on traditional education models, difficult to be changed. Self-regulated learning is a model that outlines the importance of effective planning, execution, and review strategies to foster efficient learning, including the development of meta-strategies that requires knowledge of the nature and benefit of each component and an ability to use each when appropriate. Examples of such strategies include goal setting (planning), mental rehearsal (execution), and error detection (review). The TELMI project seeks to engage with and enhance processes of learning the violin. The main aim of the project is to study how we learn musical instruments, taking the violin as a case study, from a pedagogical and scientific perspective and to create new interactive, assistive, self-learning, augmented-feedback, and social-aware systems complementary to traditional teaching. While the audio, video, and motion-capture capabilities of the TELMI system can naturally drive the review of practice activates, the data collected by the TELMI system offers the potential to support and guide planning and execution strategies to increase engagement with and utility of the system.

\section{CONCEPT}

This practice work consists of a demontration of the technologies developed by the EU-H2020-ICT Project TELMI. These enable automatic analysis of a violin performance by real-time computation of motoric and auditory features. Moreover, the player is provided with feedback to support her in training her skills. Data is captured by means of microphones and range-imaging devices.

Movement features are distinguished in low- and mid-level features, according to [1]. Low-level features include kinematics of the 3D points of the Kinect skeleton, head and overall movement direction, kinetic energy, openness, leaning of the posture on the frontal and sagittal plane, and postural tension. Mid-level features include dynamic of postural openness, suddenness, sway, and coordination (see Figure 1). Audio features concern intonation (pitch accuracy and stability at single note level, and pitch linear regression and pitch deviation at phrase/piece level), rhythm (accuracy of onsets), dynamics, articulation (recognition of specific bow-strokes, quality of attacks, transitions, sustains, and releases), and timbral properties (timbre stability, timbre richness, and tone qualities).

Feedback is provided by means of a collection of visualizations. For example, feedback on intonation consists of the performed pitch curve (see Figure 2) displayed over a piano roll representation of the music score, pr provided over the score itself (see Figure 3): for each played note, the note head is colored in green or red depending on whether the user performed it in or out of tune.

Technologies are implemented in three major components: the EyesWeb XMI Platform [3]; ViolinRT [2], a real-time play-along app; and the Planner representing the main student interface.

In the demonstration, attendants will try the technologies themselves by performing some basic tests of violin skills, and by getting an immediate feedback on their performance. 


\section{REFERENCES}

[1] Antonio Camurri, Gualtiero Volpe, Stefano Piana, Maurizio Mancini, Radoslaw Niewiadomski, Nicola Ferrari, and Corrado Canepa. 2016. The dancer in the eye: towards a multi-layered computational framework of qualities in movement. In Proceedings of the 3rd International Symposium on Movement and Computing, Thessaloniki, Greece, July 05 - 06, 2016. 6.

2] Sergio Giraldo, Rafael Ramirez, George Waddell, and Aaron Williamon. 2017. A Real-time Feedback Learning Tool to Visualize Sound Quality in Violin Performances. In Proceedings of the 10th International Workshop on Machine Learning and Music, Barcelona, Spain, October 06, 2017. 19-24.

[3] Gualtiero Volpe, Paolo Alborno, Antonio Camurri, Paolo Coletta, Simone Ghisio, Maurizio Mancini, Radoslaw Niewiadomski, and Stefano Piana. 2016. Designing Multimodal Interactive Systems using EyesWeb XMI. In Proceedings of the First International Workshop on Smart Ecosystems cReation by Visual dEsign co-located with the International Working Conference on Advanced Visual Interfaces (AVI 2016), Bari, Italy, June 07, 2016. 49-56. 